\title{
Role of non-fluorescent chromophores in inner filter effect correction and PARAFAC decomposition
}

\author{
Elfrida M. Carstea ${ }^{1 *}$, Stéphane Mounier ${ }^{2}$, Roland Redon², Cristina L. Popa ${ }^{1}$ \\ ${ }^{1}$ National Institute of R\&D for Optoelectronics, Atomistilor 409, 077125, Magurele, Romania; \\ elfrida.carstea@inoe.ro, cristina.popa@inoe.ro
}

${ }^{2}$ Univ Toulon, Aix Marseille Univ., CNRS/INSU, IRD, MIO UM 110, Mediterranean Institute of Oceanography, La Garde, France mounier@univ-tln.fr; roland.redon@univ-tln.fr

\begin{abstract}
Numerous studies have shown the impact of inner filter effect (IFE) on the fluorescence signal. IFE reduces the fluorescence intensity and distorts the fluorescence peak shape and position, through the absorption of the emitted radiation by the sample components. In this study, we aimed to understand the role of a non-fluorescing chromophore in IFE correction and PARAFAC decomposition. Solutions of three fluorophores, tryptophan, fluorescein and quinine sulfate, and an absorbing compound, green ink, have been prepared using the controlled dilution approach (CDA). PARAFAC identified three components associated with quinine sulfate, fluorescein and an IFE artifact, which was caused by a shift in peak position. Results showed that the absorption of the chromophore plays an important role in component determination. We observed that the CDA-PARAFAC was able to correct the quinine sulfate and fluorescein signals, and to suppress the IFE artifact component. However, the method was not effective in removing the IFE impact at high concentrations. The results have significant implications on the analysis of samples that contain complex mixtures of fluorophores and chromophores, such as colored natural organic matter or nutrients, like $\mathrm{NO}_{3}{ }^{2-}$.
\end{abstract}

Keywords: Inner filter effect, fluorescence spectroscopy, controlled dilution approach, PARAFAC

Corresponding author: Elfrida Carstea, National Institute of R\&D for Optoelectronics, INOE 2000, Atomistilor 409, Magurele, Ilfov, 077125; Tel: 40-31-405 07 96; e-mail: elfrida.carstea@inoe.ro

\section{Introduction}

Fluorescence spectroscopy has found several applications in environmental science for water quality monitoring of surface water, ground water, untreated and treated drinking and 
wastewater [1]. The technique has been intensively used due to its advantages such as fast measurement, no reagents needed and high sensitivity [2,3]. However, issues have been encountered with its application as several factors can change the fluorescence response. These factors include: the inner filter effect (IFE), temperature, $\mathrm{pH}$ or the presence of metal ions [2]. Among these factors, the IFE is the most severe as it can impact the shape of the spectra, reduce the fluorescence intensity and change the position of the peak, making the spectra more difficult to correct [4-7]. The approaches used to correct the IFE, for example absorption- and dilution-based methods, are relatively impractical. The absorption-based method often requires another instrument, while the dilution approach reduces the signal to noise ratio and may contaminate the sample [4,8,9]. Another approach includes the use of specific cuvette configurations to increase the path length, during measurements [7]. However, this method depends on the type of instrument or analyst [7]. The controlled dilution approach (CDA), involving a series of low dilution factors, was proposed by Luciani et al. [8] as a fast and safe solution to IFE correction. Combined with Parallel Factor Analysis (PARAFAC), it can turn into a powerful tool to avoid error and misinterpretation of fluorescence data [8].

In the past decade, PARAFAC has been commonly applied to analyze fluorescence spectra of water organic matter [10]. PARAFAC decomposes excitation-emission matrices (EEMs) into underlying chemical components [11]. Since IFE can distort EEMs, issues arise with PARAFAC decomposition of uncorrected EEMs [11]. For instance, Luciani et al. [8] found the worst results at PARAFAC decomposition on IFE uncorrected EEMs, when measuring overlapping sample components. Several studies have been made so far to show the role of various fluorophores in producing IFE and the optimum method of correcting IFE $[4,5,8,12-$ 15]. However, no study was made so far to assess how well the IFE can be corrected, with the CDA-PARAFAC approach, when a non-fluorescent chromophore is present in a solution.

In this study, we aim to understand the role of a non-fluorescent chromophore in IFE correction and PARAFAC decomposition. We applied the CDA-PARAFAC on EEMs recorded for solutions of three fluorophores and one chromophore. We also tested if the approach can be used at different concentration levels.

\section{Methods and materials}

\subsection{Sample preparation}


Solutions of fluorescein, quinine sulfate and tryptophan were prepared in $0.1 \mathrm{M} \mathrm{H}_{2} \mathrm{SO}_{4}$ (Aldrich). All chemicals were analytically graded. The fluorophores were chosen due to their high quantum efficiency and peak overlapping characteristics [16]. Fluorophore solutions were prepared in mixtures of $0 \%, 33 \%, 66 \%$ and $100 \%$ of an initial concentration of fluorescein $\left(\mathrm{F}_{0}, 75 \mathrm{mg} / \mathrm{L}\right)$ and quinine sulfate $\left(\mathrm{QS}_{0}, 250 \mathrm{mg} / \mathrm{L}\right)$, while tryptophan $(170 \mathrm{mg} / \mathrm{L})$ represented $33 \%$ of the total analyzed volume (Table A1). To each mixture, commercially available green ink was added as an absorbing component, in concentrations of $0 \%, 10 \%$ and $100 \%$, corresponding to maximum absorption peak values of $0,0.1$ and 1 . A total of 45 solutions were obtained.

Subsequently, these solutions were diluted to apply the CDA correction. Details regarding the CDA methodology can be found in Luciani et al. [8]. The CDA correction takes into account the fluorescence intensity of the undiluted sample $\left(I_{0}\left(\lambda_{e x}, \lambda_{e m}\right)\right)$, the intensity of the diluted sample $\left(I_{1}\left(\lambda_{e x}, \lambda_{e m}\right)\right)$ and the dilution factor $(p)$, as shown in Eq. (1): Corrected EEM $=\left(\frac{p \cdot I_{1}\left(\lambda_{\text {ex/em }}\right)}{I_{0}\left(\lambda_{\text {ex/em }}\right)}\right) \frac{1}{p-1}$

Dilution factors from 2 to 16 were used on the 45 solutions, by mixing them with $0.1 \mathrm{M} \mathrm{H}_{2} \mathrm{SO}_{4}$. (Fig. A1), based on Eq. (2):

$$
D_{1}=2 * D_{2}=8 * D_{3}=16 * D_{4}
$$

The concentrations D1 and D2 corresponded to a high IFE, while D3 and D4 corresponded to the quasilinear part of the IFE curve. Reference dilutions were prepared to represent the solutions with no IFE and physico-chemical changes [8]. The reference solutions were obtained by mixing $100 \mu \mathrm{L}$ of initial solution to $1,900 \mu \mathrm{L}$ of $0.1 \mathrm{M} \mathrm{H}_{2} \mathrm{SO}_{4}$.

\subsection{Measurements}

The absorption spectra were registered, within the range of 200 to $800 \mathrm{~nm}$, with a Shimadzu1800 spectrometer using $1 \mathrm{~cm}$ quartz cell. The EEMs were acquired with a HITACHI F4500 fluorimeter. The EEMs were measured in the emission wavelength range of 200-600 nm (slit $2.5 \mathrm{~nm}$ ) and in the excitation wavelength range of 200 to $550 \mathrm{~nm}$ (slit $2.5 \mathrm{~nm}$ ), every $5 \mathrm{~nm}$. The PM voltage was fixed at $700 \mathrm{~V}$ and the scan speed at 2,400 $\mathrm{nm} / \mathrm{min}$ (response $0.1 \mathrm{~s}$ ). The absorption and fluorescence spectra of each component are presented in Figure A3. The CDA-PARAFAC analysis was detailed by Luciani et al. [8]. Four groups of EEMs were considered for CDA-PARAFAC correction: the EEMs group measured on reference 
solutions, the uncorrected EEM group, the absorption corrected EEM and the CDA corrected EEMs. These groups represented the four 3-way tensors with the dimensions 7 x 46 x 51, as detailed by Luciani et al. [8]. Before the CDA-PARAFAC procedure Raman and Rayleigh scattering were removed from the EEMs, according to the method of Zepp et al. [17],. CP/PARAFAC algorithm, with “progmeef” Graphical User Interface developed by R. Redon (http://woms18.univ-tln.fr/progmeef/) was used for EEM decomposition. The number of components was selected when we obtained the higher component number with a core consistency score above 60\%, according to Mounier et al. [18].

\section{Results and discussions}

\subsection{Fluorescence PARAFAC components}

Three PARAFAC components were obtained (Fig. 1). Component $1\left(C_{1}\right)$ belonged to quinine sulfate, with excitation and emission wavelengths at 250 \& $350 / 430 \mathrm{~nm}$. The second component $\left(\mathrm{C}_{2}\right)$ was attributed to fluorescein, at $\lambda_{\text {ex/em }}=440 / 510 \mathrm{~nm}$. The third component $\left(\mathrm{C}_{3}\right), \lambda_{\mathrm{ex} / \mathrm{em}}=350 / 460 \mathrm{~nm}$, was believed to be an artifact, as it could not be attributed to any of the substances that were used in the mixtures. $\mathrm{C}_{3}$ reflected the impact of the IFE on PARAFAC decomposition. The IFE causes a peak shift to shorter or longer wavelengths, depending on component aggregation and solutions composition [5,19]. In the present analysis, the IFE peak position change determined PARAFAC to extract a false third component. Yu et al. [20] also found that component peak shift undermined the global PARAFAC model.

PARAFAC was not able to extract tryptophan. Tryptophan fluorescence intensity may have been quenched by the fluorophores or chromophore.. Galinha et al. [12] andWang et al. [21] proved that tryptophan-like fluorescence intensity may be quenched by other components competing for similar excitation wavelengths or by complex formation with other components present in the solution. Another explanation may be that the concentration of tryptophan was constant in the solutions and could not be identified as an individual component. 
Fig. 1 PARAFAC decomposition of EEMs of fluorescing and absorbing compounds mixtures. $C_{1}-$ quinine sulfate; $C_{2}$ - fluorescein; $C_{3}-$ IFE artifact.

\subsection{Chromophore impact on PARAFAC decomposition}

The fluorescence response of $C_{1}$ (quinine sulfate) and $C_{2}$ (fluorescein), at each dilution and chromophore concentration, is presented in Figures 2 and 3. Results showed that only the low dilutions, $D_{3}$ and $D_{4}$, preserved the linearity of $C_{1}$ fluorescence response; however, the chromophore substantially reduced the fluorescence response of $\mathrm{C}_{1}$. In addition, the fluorescence intensity of quinine sulfate decreased with the chromophore increasing concentration. The fluorescence intensity of $\mathrm{D}_{3}$ was higher compared to $\mathrm{D}_{4}$, at the solutions with no chromophore added. However, when the maximum quantity of chromophore was added to the solution, both $\mathrm{D}_{3}$ and $\mathrm{D}_{4}$ dilutions displayed the same fluorescence intensity. This finding proved that chromophores can have a substantial impact on the fluorescence signal, even at low fluorophore concentrations, but that the impact is greater at higher concentrations. The fluorescence intensity decreased with $\sim 44 \%$, at $\mathrm{D}_{4}$ dilution, from the the solution with no chromophore, to the solution with maximum concentration of chromophore. While a $\sim 68 \%$ decrease was observed at $\mathrm{D}_{3}$, at maximum chromophore concentration. Also, the addition of the maximum concentration of chromophore, reduced the fluorescence intensity of $\mathrm{D}_{2}$ with $\sim 67 \%$. The highest reduction of fluorescence intensity was seen at $\mathrm{D}_{1}$, of $\sim 80 \%$.

Fig. 2 The fluorescence intensity of quinine sulfate, at each dilution, in relation to the chromophore. 
Fig. 3 The fluorescence intensity of fluorescein, at each dilution, in relation to the chromophore.

Similar results were detected for the fluorescein component. We recorded a linear response for $\mathrm{D}_{3}$ and $\mathrm{D}_{4}$ (Fig. 3), and a non-linear response for $\mathrm{D}_{1}$ and $\mathrm{D}_{2}$. Also, the fluorescence intensity decreased substantially when the highest concentration of chromophore was added to the solution. Solution $\mathrm{D}_{4}$ showed a $\sim 42 \%$ decrease, $\mathrm{D}_{3}$ a $\sim 64 \%$ decrease, $\mathrm{D}_{2} \mathrm{a} \sim 50 \%$ decrease and $\mathrm{D}_{1}$ showed a $78 \%$ decrease in fluorescence intensity, from the solution with no chromophore added. Unlike quinine sulfate, fluorescein dilutions $\mathrm{D}_{1}$ and $\mathrm{D}_{2}$ displayed the same fluorescence intensity at the solution with $10 \%$ chromophore and with no chromophore. According to Murphy et al. [11], IFE is most severe at short wavelengths. Quinine sulfate has a higher absorption at shorter wavelengths compared to fluorescein (Fig. A1), indicating that quinine sulfate could be more sensitive to IFE at low chromophore concentrations. In addition, the absorption spectra of quinine sulfate and fluorescein overlap, resulting in further IFE.

The third component, $C_{3}$, attributed to the IFE, presented no signal at dilutions $\mathrm{D}_{3}$ and $\mathrm{D}_{4}$ because there was no IFE impact at these low concentrations. For dilutions $D_{1}$ and $D_{2}$, the component presented an ascending trend with each concentration (Fig. 4). The fluorescence intensity of $\mathrm{D}_{2}$ solutions was similar at all chromophore concentrations. However, the fluorescence intensity was substantially lower at $\mathrm{D}_{1}$ solution, with maximum chromophore concentration, compared to $\mathrm{D}_{1}$ solutions with the other chromophore concentrations (10\% and $0 \%$ ). The fluorescence signal of $\mathrm{C}_{3}$ contrasted the response of quinine sulfate and fluorescein, suggesting that $\mathrm{C}_{3}$ values represented the reduction in fluorescence intensity due to IFE, which resulted in linearity loss, at dilutions $D_{1}$ and $D_{2}$ of quinine sulfate and 
fluorescein. This suggested that PARAFAC may identify the IFE induced by fluorophores but not the effect of chromophores, because that trilinear part of PARAFAC composition is null (no fluorescence response).

Fig. 4. The response of the PARAFAC component associated with IFE in relation to chromophore concentration.

\subsection{CDA correction of components}

The correction was applied to the combined EEMs dataset of $\mathrm{D}_{1}$ and $\mathrm{D}_{2}$, representing high IFE, and to the dataset of $\mathrm{D}_{3}$ and $\mathrm{D}_{4}$, for low IFE. Based on the $60 \%$ rule of core consistency provided by Mounier et al. [18], we obtained the best decomposition at 4 components, for the $\mathrm{D}_{1}$ and $\mathrm{D}_{2}$ dataset, and at only 3 components, for the $\mathrm{D}_{3}$ and $\mathrm{D}_{4}$ dataset (Fig. A4). However, the decomposition was forced to three components for better comparison between datasets, and because the fourth component, in the D1 and D2 dataset, was most likely another artifact produced by the IFE.

Figure 5 displays the CDA correction of quinine sulfate and fluorescein solutions, for the $\mathrm{D}_{1}$ and $\mathrm{D}_{2}$ dataset. CDA successfully established linearity for fluorescein and provided the same fluorescence intensity for each solution, irrespective of chromophore concentration. However, the CDA did not entirely remove the IFE impact on the solutions with the highest concentrations of quinine sulfate and chromophore. At the maximum concentration of quinine sulfate and chromophore, a difference of $38 \%$ of fluorescence intensity from the solution with no chromophore was observed. This was potentially caused by the high susceptibility to 
IFE impact of quinine sulfate, compared to fluorescein. $C_{3}$ was completely suppressed at all chromophore concentrations.

For low concentrations, $\mathrm{D}_{3}$ and $\mathrm{D}_{4}$, the linearity of the signal was completely restored at both components, $\mathrm{C}_{1}$ and $\mathrm{C}_{2}$ (Fig. $5 \mathrm{~b}$ ). The CDA correction for $\mathrm{C}_{3}$ displayed a flat signal noise.

Fig. 5 CDA correction of quinine sulfate, fluorescein and IFE artifact (C3) for a) $D_{1}$ and $D_{2}$, and b) $D_{3}$ and $D_{4}$ datasets.

\section{Conclusions}

In this study, we presented the application of CDA correction on a complex solution of three fluorophores and a chromophore, and the impact of IFE on PARAFAC decomposition. The IFE reduced the fluorescence intensity and produced a fluorescence peak shift, which was identified by PARAFAC as a separate component. In the presence of the chromophore, CDA correction was able to restore the quinine sulfate and fluorescein signals to linearity and to completely suppress the artifact component associated with IFE. Tryptophan signal was not identified by PARAFAC, even after CDA correction. Moreover, the correction method was not sufficiently effective at high concentrations of fluorophore and chromophore. These results have significant implications on the analysis of samples that contain a complex mixture of relatively unknown fluorophores and chromophores, such as the natural organic matter or nutrients, like $\mathrm{NO}_{3}{ }^{2-}$. Particularly, we recommend to use CDA or absorption correction, systematically, before EEM decomposition. Future studies are needed to find a method of extracting the non-fluorescent compounds, at the same time with fluorescing ones, and to enhance the IFE correction and detection at short wavelengths. 


\section{Acknowledgements}

E.M. Carstea acknowledges the financial support of the Université de Toulon for laboratory experiment. E.M. Carstea and C.L. Popa also acknowledge the support of the Ministry of Research and Innovation, CNCS-UEFISCDI, project number PN-III-P1-1.1-TE-2016-0646, within PNCDI III and project number 18N/2019, under the Core Program OPTRONICA VI.

\section{References}

[1] E.M. Carstea, J. Bridgeman, A. Baker, D.M. Reynolds, Fluorescence spectroscopy for wastewater monitoring: A review, Water Res. 95 (2016) 206-219. doi:10.1016/j.watres.2016.03.021.

[2] R.K. Henderson, A. Baker, K.R. Murphy, A. Hambly, R.M. Stuetz, S.J. Khan, Fluorescence as a potential monitoring tool for recycled water systems: A review, Water Res. 43 (2009) 863-881. doi:10.1016/j.watres.2008.11.027.

[3] L. Yang, J. Hur, W. Zhuang, Occurrence and behaviors of fluorescence EEMPARAFAC components in drinking water and wastewater treatment systems and their applications: A review, Environ. Sci. Pollut. Res. 22 (2015) 6500-6510. doi:10.1007/s11356-015-4214-3.

[4] D.N.. Kothawala, K.R.. Murphy, C.A.. Stedmon, G.A.. Weyhenmeyer, L.J.. Tranvik, Inner filter correction of dissolved organic matter fluorescence, Limnol. Oceanogr. Methods. 11 (2013) 616-630. doi:10.4319/lom.2013.11.616.

[5] M. Tarai, A.K. Mishra, Inner filter effect and the onset of concentration dependent red shift of synchronous fluorescence spectra, Anal. Chim. Acta. 940 (2016) 113-119. doi:10.1016/j.aca.2016.08.041.

[6] A. Kasparek, B. Smyk, A new approach to the old problem: Inner filter effect type I and II in fluorescence, Spectrochim. Acta Part A Mol. Biomol. Spectrosc. 198 (2018) 297-303. doi:10.1016/J.SAA.2018.03.027.

[7] S.K. Panigrahi, A.K. Mishra, Study on the dependence of fluorescence intensity on optical density of solutions: the use of fluorescence observation field for inner filter effect corrections, Photochem. Photobiol. Sci. 18 (2019) 583-591. doi:10.5860/choice.51-2070.

[8] X. Luciani, S. Mounier, R. Redon, A. Bois, A simple correction method of inner filter effects affecting FEEM and its application to the PARAFAC decomposition, Chemom. 
Intell. Lab. Syst. 96 (2009) 227-238. doi:10.1016/j.chemolab.2009.02.008.

[9] J.E. Cohen, P. Comon, X. Luciani, Correcting inner filter effects, a non multilinear tensor decomposition method, Chemom. Intell. Lab. Syst. 150 (2016) 29-40. doi:10.1016/j.chemolab.2015.11.002.

[10] K.R. Murphy, C. a Stedmon, P. Wenig, R. Bro, OpenFluor- an online spectral library of auto-fluorescence by organic compounds in the environment, Anal. Methods. 6 (2014) 658-661. doi:10.1039/c3ay41935e.

[11] K.R. Murphy, C.A. Stedmon, D. Graeber, R. Bro, Fluorescence spectroscopy and multi-way techniques. PARAFAC, Anal. Methods. 5 (2013) 6557-6566. doi:10.1039/c3ay41160e.

[12] C.F. Galinha, G. Carvalho, C.A.M. Portugal, G. Guglielmi, M.A.M. Reis, J.G. Crespo, Two-dimensional fluorescence as a fingerprinting tool for monitoring wastewater treatment systems, J. Chem. Technol. Biotechnol. 86 (2011) 985-992. doi:10.1002/jctb.2613.

[13] C. Goletz, M. Wagner, A. Grübel, W. Schmidt, N. Korf, P. Werner, Standardization of fluorescence excitation-emission-matrices in aquatic milieu, Talanta. 85 (2011) 650656. doi:10.1016/j.talanta.2011.04.045.

[14] T. Larsson, M. Wedborg, D. Turner, Correction of inner-filter effect in fluorescence excitation-emission matrix spectrometry using Raman scatter, Anal. Chim. Acta. 583 (2007) 357-363. doi:10.1016/j.aca.2006.09.067.

[15] A. Mendonça, A.C. Rocha, A.C. Duarte, E.B.H. Santos, The inner filter effects and their correction in fluorescence spectra of salt marsh humic matter, Anal. Chim. Acta. 788 (2013) 99-107. doi:10.1016/j.aca.2013.05.051.

[16] X. Luciani, R. Redon, S. Mounier, How to correct inner filter effects altering 3D fluorescence spectra by using a mirrored cell, Chemom. Intell. Lab. Syst. 126 (2013) 91-99. doi:10.1016/j.chemolab.2013.04.014.

[17] R.G. Zepp, W.M. Sheldon, M.A. Moran, Dissolved organic fluorophores in southeastern US coastal waters: correction method for eliminating Rayleigh and Raman scattering peaks in excitation-emission matrices, Mar. Chem. 89 (2004) 15-36. doi:https://doi.org/10.1016/j.marchem.2004.02.006.

[18] S. Mounier, H. Zhao, C. Garnier, R. Redon, Copper complexing properties of dissolved 
organic matter: PARAFAC treatment of fluorescence quenching, Biogeochemistry. 106 (2011) 107-116. doi:10.1007/s10533-010-9486-6.

[19] H. Li, YuzhuHu, Spectroscopic investigation of inner filter effect by magnolol solutions, Spectrochim. Acta - Part A Mol. Biomol. Spectrosc. 68 (2007) 1263-1268. doi:10.1016/j.saa.2007.02.002.

[20] H. Yu, H. Liang, F. Qu, Z. Han, S. Shao, H. Chang, G. Li, Impact of dataset diversity on accuracy and sensitivity of parallel factor analysis model of dissolved organic matter fluorescence excitation-emission matrix., Sci. Rep. 5 (2015) 10207. doi:10.1038/srep10207.

[21] Z. Wang, J. Cao, F. Meng, Interactions between protein-like and humic-like components in dissolved organic matter revealed by fluorescence quenching, Water Res. 68 (2015) 404-413. doi:10.1016/j.watres.2014.10.024. 